\title{
Estimation of the Aerobic-anaerobic Transition by Heart Rate Variability in Athletes and Non-athletes Subjects
}

\author{
Antonio López-Fuenzalida (Corresponding author) \\ Carrera de Kinesiología, UDA Cs. de la Salud, Facultad de Medicina, Pontificia Universidad Católica de Chile, Av. \\ Vicuña Mackenna 4860, Macul, 7820436, Santiago, Chile \\ E-mail: aelopezf@uc.cl \\ David Laroze N. \\ Instituto de Alta Investigación, Universidad de Tarapacá, Chile, Casilla 7D, Arica, Chile \\ E-mail: dlarozen@uta.cl \\ Francisco José Berral de la Rosa \\ Departamento de Deporte e Informática, Universidad Pablo Olavide, España, Carretera Utreras Km 1, 41013, Sevilla, España \\ E-mail: fjberde@upo.es \\ Jorge Cancino L. \\ Escuela de Kinesiología, Facultad de Medicina, Universidad Mayor, Chile \\ E-mail: jorge.cancino@umayor.cl
}

Received: 28-01- 2016

doi:10.7575/aiac.ijkss.v.4n.2p.36
Accepted: 02-04- 2016

Published: 30-04- 2016

URL: http://dx.doi.org/10.7575/aiac.ijkss.v.4n.2.p.36

This research was partially supported from Basal Program Center for Development of Nanoscience and Nanotechnology (CEDENNA) and UTA-project 8750-12.

\begin{abstract}
Background: The estimate of aerobic-anaerobic threshold is commonly used for exercise prescription in athletes and non-athletes subjects; however, the methods currently used are expensive, and some of them use invasive techniques. The analysis of changes in heart rate variability during exercise may contribute to the introduction of a novel methodology to estimate the aerobic-anaerobic metabolic transition. Objective: The purpose of this study was to estimate the heart rate variability threshold (HRVT) through the Discrete Wavelet Transform (DWT) and compare the exercise intensities at which this happens to the moment when the aerobic-anaerobic metabolic transition occurs, estimated by the ventilatory threshold 2 (VT2) in athletes and non-athletes. Methods: 24 male subjects were enrolled (12 athletes; 12 non-athletes). Ventilatory parameters and R-R intervals were recorded breath by breath in a maximal incremental intensity exercise. HRVT was estimated through DWT and the VT2 was determined by 5 indicators: respiratory quotient $\geq 1.0$, non-linear increase of the $\mathrm{VE}$ and $\mathrm{VCO}_{2}$, decrease of the $\mathrm{P}_{\mathrm{ET}} \mathrm{CO}_{2}$ and increase of the $\mathrm{P}_{\mathrm{ET}} \mathrm{O}_{2}$. Reserve heart rate frequency percentages (\%RHRF) are determined, compared and correlated to VT2 (\%RHRF-VT2) and HRVT (\%RHRF-HRVT). We used a significance level of $\mathrm{p}<0.05$ for all our analysis. Results: The results showed that there is no significant difference between the \%RHRF-VT and \%RHRF-HRVT in the assessed subject groups (total, athletes, non-athletes). There is a correlation between \%RHRF-VT and \%RHRF-HRVT in the whole group $(\mathrm{r}=0.91 ; \mathrm{p}<0.001)$, athletes $(\mathrm{r}=0.84 ; \mathrm{p}<0,001)$ and non-athletes $(\mathrm{r}=0.88 ; \mathrm{p}<0,001)$. Conclusion: We conclude that the aerobic-anaerobic metabolic transition (i.e. VT2) during an incremental maximal exercise can be estimated through the HRVT measured by the DWT in athletes and non-athletes.
\end{abstract}

Keywords: non-linear analysis, anaerobic threshold, exercise test

\section{Introduction}

The aerobic-anaerobic metabolic transition (AAMT) that is estimated throughout the use of the ventilatory parameters in a maximal incremental intensity exercise is one of the most used methods to determine the physical work load in aerobic training processes in athletes and non-athletes people (Knoepfli et al., 2004; Marks \& Lightfoot, 1999). This allows estimate work loads based on a close knowledge about the predominant metabolism in the person while he or she is doing physical activity, contributing in this way to achieve the planned goals by the professionals of the physical activity, sports and health field (Meyer, Lucia, \& Earnest, 2005). However, determining the AAMT is usually an expensive method what means a low level of access for a large part of the population, making this process of assesment almost impossible to massify due to lack of resources. This limits the estimation of this metabolic parameter of great 
importance when prescribing exercise. Because of this, it is necessary to use some other assesment and analysis resources of biological signaling which allow accurately identify this key threshold in exercise prescription.

It is in this context where heart rate variability (HRV) has become more important in the last time, understanding it as an analysis tool for cardiovascular variability that studies fluctuations between heartbeats expressed in the R-R intervals of the electrocardiogram (ECG) that has to be understood as an indicator of the integration of the autonomous nervous system responses given by several organs and systems when facing different stimuli (physical, pharmacological (Elghozi, Girard, \& Laude, 2001) or psychological (Aubert, Seps, \& Beckers, 2003; Drago et al., 2007; Sartor, Vailati, Valsecchi, Vailati, \& La Torre, 2013; Thayer \& Sternberg, 2006)). This represents an integrated response to neural, humoral and self-regulation influences (Accurso, Shamsuzzaman, \& Somers, 2001; Dewey et al., 2007; R. Freeman, 2006).

However, when using HRV in conditions where the ECG signal does not present stationary characteristics like physical activity performance with incremental loads (usually used in an AAMT estimation test), it has been identified that the spectral and temporal HRV analysis would have a difficulty describing the changes in the autonomic function (Pichot et al., 1999; Sone, Yamazaki, Fujii, Fukuoka, \& Ikegami, 1996). On the other hand, the non-linear and spectro-temporal methods have shown their utility in this field, highlighting the Poincaré plotting (de la Cruz Torres, López, \& Orellana, 2008) and the Discrete Wavelet Transform (DWT) (Verlinde, Beckers, Ramaekers, \& Aubert, 2001). The latest is considered as the most accurate tool to identify HRV changes under these conditions (Belova, Mihaylov, \& Piryova, 2007; Verlinde et al., 2001). Given the cost and methodology benefits that HRV presents and considering DWT as one of the best methods to estimate HRV changes in non-stationary states, the present study aims to estimate the HRV threshold through DWT and compare the intensity of the exercise where the ventilatory threshold 2 (VT2) occurs in athletes and non-athletes.

\section{Methods}

\subsection{Experimental Approach to the Problem}

We examined the ability of HRV as a predictor of the aerobic-anaerobic metabolic transition (AAMT) during the performance of a maximal incremental test in athletes and non-athletes. For this purpose, two groups were formed with young male athletes $(\mathrm{n}=12)$ and non-athletes $(\mathrm{n}=12)$. Both groups executed a maximal incremental test until volitional exhaustion, where the HRV and the AAMT were determined. This is a non-experimental, descriptive crosssectional research study with a quantitative approach.

\subsection{Participants}

Twenty-four male subjects were considered in the assessment processes, who were divided in an athlete group (SG; $\mathrm{n}=$ 12; age $21.8 \pm 2.4 \mathrm{y}$; height $176.2 \pm 10.4 \mathrm{~cm}$; body mass: $78.6 \pm 11.5 \mathrm{~kg}$ ) and a non-athlete group (NSG; $\mathrm{n}=12$; age $22.2 \pm 2.2 \mathrm{y}$; height $176.9 \pm 4.1 \mathrm{~cm}$; body mass $73.3 \pm 10.1 \mathrm{~kg}$ ). The SG was composed of Chilean national team members of rugby $(n=5)$, taekwon-do $(n=3)$ and volleyball $(n=4)$. Inclusion criteria were: i) absence of cardiac, respiratory or metabolic pathologies; ii) absence of traumatological and/or orthopedic dysfunctions pathologies. Participants were asked to not perform any physical activities or consumed any drug 24 hours previous to the assessments, and not have eaten or drunk any food or stimulating drinks 4 hours before the assessments.

The study was approved by the Ethics Committee of Córdoba University (Spain), ascribing entirely to the Helsinki Declaration. All participants signed an informed consent prior to participating in this study. In this consent, all risks and benefits of the participation in this study were described. We comply with the human and animal experimentation policy statements guidelines of the American College of Sport Medicine. Sample size was computed according to the $r$ $=0.82$ correlation between the HRV thresholds (determined by a temporary method) and ventilatory threshold reported by Karapetian et al. (Karapetian, Engels, \& Gretebeck, 2008) in a group of healthy adults. A total of 11 participants per group would yield a power of $90 \%$ and $\alpha=0.05$.

\subsection{Testing Procedures}

We considered the application of a physical activity protocol of incremental maximal intensity in a treadmill (Treadmill PowerJog Cardio Sport) with a constant speed of $6.4 \mathrm{~km} / \mathrm{h}$ with an initial tilt of $0 \%$, with a slope increase of $4 \%$ every 2 minutes until volitional fatigue. Beat to beat R-R intervals are recorded throughout the whole exercise phase with a s810i Polar heart rate frequency monitor (Polar Electro, Kempele, Finland) (Cottin et al., 2007; Karapetian et al., 2008). Ventilatory parameters $\mathrm{P}_{\mathrm{ET}} \mathrm{O}_{2}, \mathrm{P}_{\mathrm{ET}} \mathrm{CO}_{2}, \mathrm{VE}, \mathrm{VCO}_{2}, \mathrm{VO}_{2}, \mathrm{VE} / \mathrm{VO}_{2}$ and $\mathrm{VE} / \mathrm{VCO}_{2}$ are recorded breath by breath with an ergospirometer (Sensor Medics, Vmax 29 Series; face mask, Hans Rudolph, medium and small, 7930 series). All assessments were done in the Exercise Physiology Laboratory of the Medicine Faculty of Universidad Mayor in Santiago, Chile.

\subsection{HRV threshold (HRVT) estimation method.}

R-R intervals of the incremental maximal intensity phase were used for the HRV analysis. Data was automatically filtered to remove premature or lost heartbeats. An R-R interval was interpreted as premature if it deviated over $30 \%$ from the previous qualified interval (Karapetian et al., 2008). To obtain the HRVT, a DWT was developed because this allows to decompose the signal in a way to analyze the HRV and its variance, detect fractal patterns, detect particular zones of behaviors, eliminate low frequency components (suppressing the signal tendency); eliminate high frequency components, obtain good adjustments or suppress noise that is not important to analyze (Manso, 2013). The DWT 
requires the use of digital filters, obtaining a step-scale representation of a digital signal, using filters of different cut-off frequencies to analyze the signal in different scales. Signal resolution changes through filter operations. The scale changes through subsampling and supersampling operations. Subsampling reduces the sample rate by eliminating some signal samples. Subsampling by an $\eta$ factor reduces $\eta$ times the number of signal samples. Supersampling increases the sample rate, adding new samples given that supersampling by an $\eta$ factor increases $\eta$ times the number of signal samples.

In this work, the R-R intervals have been analyzed through the Discrete Wavelet Transform (DWT) (Cohen, 1994). In order to define the DWT, let $\zeta(n)$ be a sequence of numbers with $n \in \mathrm{N}$; the Wavelet transform of $\zeta$ is given by:

$$
W[n]=\Sigma \zeta(n) \Psi_{\mathrm{j}, \mathrm{k}}(n),
$$

$\Psi_{\mathrm{j}, \mathrm{k}}(n)$, being a discrete wavelet defined by:

$$
\Psi_{\mathrm{j}, \mathrm{k}}(n)=2^{-\mathrm{j} / 2} \Psi\left(2^{-\mathrm{j}} \mathrm{n}-\mathrm{k}\right),
$$

where $\{j, k\}$ are parameters related to translation property and the scale changes of the DWT, respectively; these two properties provide the multire-solution structure of the DWT. Note that in the DWT there are two fundamental filters: the low pass filter, $l$, and the high pass filter, $h$, and they are connected via

$$
(-1)^{n} l(n)=h(L-1-n)
$$

therefore, when given $l$, the other is calculated automatically. Our transform is performed by doing $p$ levels of decomposition using a recursive pyramidal filter scheme. For $J$ levels of decomposition and signal length $2^{L}$, the output is in the form:

$$
\left\{A_{J},\left\{D_{J}, D_{J-1}, \ldots D_{2}, D_{1}\right\}\right\}
$$

Where $A_{J}$ is the sub-signal approximation of length $2^{L-J}$ and $D_{k}$ is the $k^{\text {th }}$ detail sub-signal of length $2^{L-k}$. We used Daubechies wavelet functions with $J=4$ and the level of decompositions are denoted by $d_{j}$ with $\mathrm{j}=(1, \ldots, 4)$. Our numerical code is written in Scilab, based on the Wavelab toolbox and the Wavelet package for the Mathematica program (WolframResearch, Inc., Mathematica, Version 10.1, Champaign, IL, 2015). More mathematical details of the Wavelet theory can be found in Ref (Cohen, 1994).

Figure 1 shows this procedure for an arbitrary subject. We enhance that the non-linear features of the signal are better exposed in $d_{4}$. Hence, in the following we will calculate the threshold using this filter with the Mathematica package. Figure 2 shows a colored-code phase diagram of the levels as a function of the steps (superior frame) and the computation of the threshold (inferior frame).

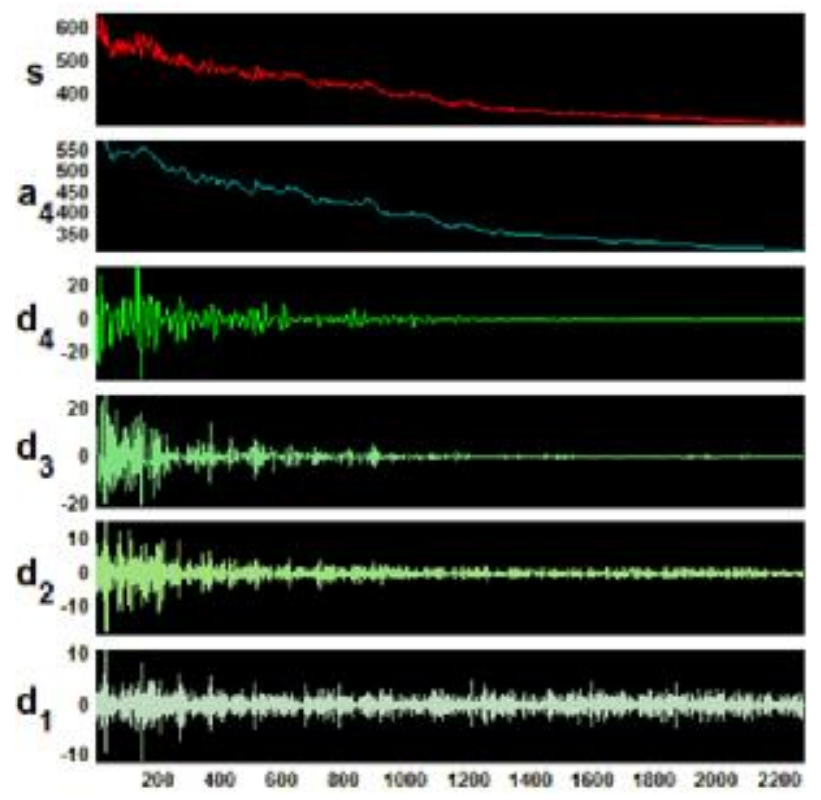

Figure 1. Representation of the application of the DWT to HRV signal in a maximal incremental intensity exercise in a given subject. 

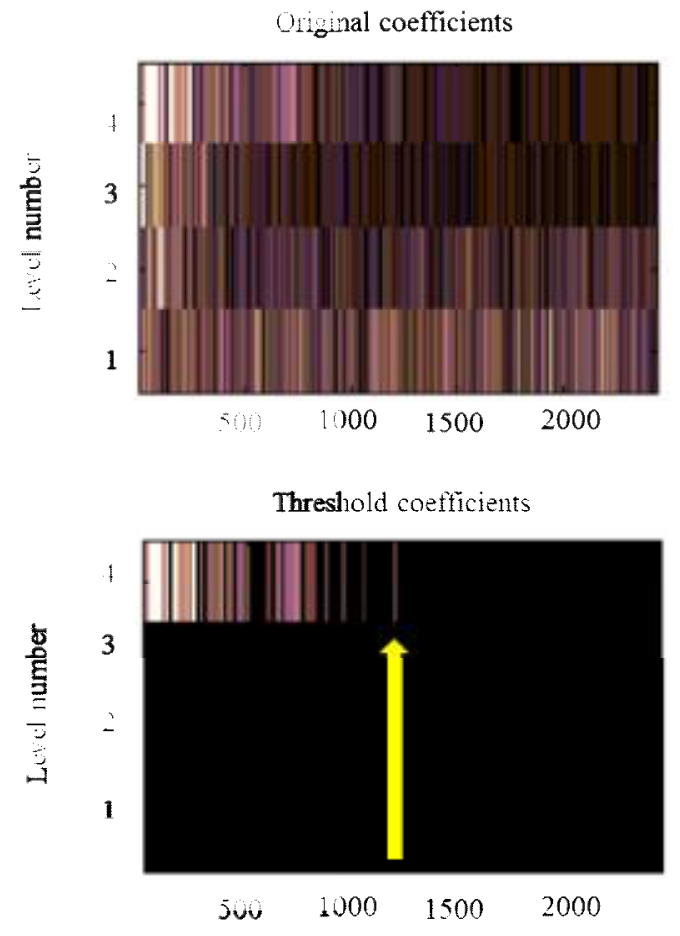

Figure 2. Colored-code phase diagram of the levels as a function of the steps (superior frame) and the computation of the threshold (inferior frame). The arrow shows the point where the signal pattern changes, representing HRVT, which occurs in a determined step in the $x$ axis.

At the same time that the HRVT was determined, the heart rate frequency was also determined, expressed as a percentage of the reserve heart rate frequency (\%RHRF) through the Karvonen equation (Karvonen, 1957), which allowed to establish the \%RHRF-HRVT.

\subsection{Methods for the estimation of the aerobic-anaerobic metabolic transition (AAMT)}

The AAMT was determined by the Ventilatory Threshold 2 (VT2) (Binder et al., 2008), through 5 ventilatory parameters during the incremental maximal test: respiratory quotient $\geq 1.0$, non-linear increase of the VE, non-linear increase of the $\mathrm{VCO}_{2}$, decrease of the $\mathrm{P}_{\mathrm{ET}} \mathrm{CO}_{2}$ and increase of the $\mathrm{P}_{\mathrm{ET}} \mathrm{O}_{2}$. At the same time that the VT2 was determined, the heart rate frequency was also determined to later express this parameter as \%RHRF through the Karvonen equation (21). This allowed to establish the \%RHRF-VT2 relation.

\subsection{Statistical Analyses}

All values are reported as mean \pm standard deviation (SD). Normality and homoscedasticity assumptions for all data were checked respectively with Shapiro-Wilk and Levene tests. To determine differences between \%RHRF-HRVT and $\%$ RHRF-VT, a paired $t$-test was used. Correlation between dependent variables was done by means of Pearson productmoment correlation coefficient. The $\alpha$ level was set at $\mathrm{p}<0.05$ for statistical significance. All statistical calculations were performed using STATA (Version 13).

\section{Results}

Table 1 shows the values of \%RHRF-HRV and \%RHRF-VT2 in the SG and NSG groups and when these are conjointly analyzed. No statistical significant differences were observed between \%RHRF-HRV and \%RHRF-VT2 in the SG or NSG group or when these were combined.

Table 1. \%RHRF-HRVT and \%RHRF-VT2 values in SG, NSG and their combination.

\begin{tabular}{lcccc}
\hline & \%RHRF-HRVT & \%RHRF-VT2 & Dif-means & P value \\
\hline SG $(\mathrm{n}=12)$ & $77.6 \pm 4.8$ & $77.9 \pm 4.3$ & 0.24 & 0.76 \\
NSG $(\mathrm{n}=12)$ & $69.7 \pm 7.6$ & $69.9 \pm 8.3$ & 0.20 & 0.86 \\
\hline TOTAL $(\mathrm{n}=24)$ & $73.7 \pm 7.4$ & $73.9 \pm 7.6$ & 0.22 & 0.74 \\
\hline
\end{tabular}

\%RHRF-HRVT: reserve heart rate frequency percentage when reaching the variability threshold of the cardiac rhythm; \%RHRF-VT2: reserve heart rate frequency percentage when reaching ventilatory threshold 2; SG: athletes; NSG: nonathletes. 
Table 2 shows the correlations between \%RHRF-VT2 and \%RHRF-HRVT in groups SG, NSG and when both are jointly analyzed. Strong and significant direct relationships were found for each one of the groups and also when analyzing both groups together, which highest correlation can be found $(r=0.91)$.

Table 2. Correlation between \%RHRF-HRVT and \%RHRF-VT2 in SG, NSG and combination

\begin{tabular}{lccc}
\hline & $\mathrm{r}$ & $\mathrm{r}^{2}$ & $\mathrm{P}$ value \\
\hline SG $(\mathrm{n}=12)$ & 0.84 & 0.70 & $<0.001$ \\
NSG $(\mathrm{n}=12)$ & 0.88 & 0.78 & $<0.0001$ \\
\hline TOTAL $(\mathrm{n}=24)$ & 0.91 & 0.82 & $<0.0001$ \\
\hline
\end{tabular}

\%RHRF-HRVT: reserve heart rate frequency percentage when reaching the variability threshold of the cardiac rhythm;

\%RHRF-VT2: reserve heart rate frequency when reaching the ventilator threshold 2; SG: athletes; NSG: non-athletes.

\section{Discussion}

The main result of our research was that the estimation of the AAMT by HRV analysis through the DWT occurs at the same intensity of the \%RHRF to which VT2 occurs, determined by ergoespirometry in athletes and non-athletes. The maximal mean difference is of $0.24 \%$ of the $\%$ RHRF between thresholds. Additionally, strong and direct correlations were found between exercise intensities, relativized through the \%RHRF, where the studied thresholds occur.

Our findings are based in the existent relationships between the Autonomous Nervous System (ANS) and the Cardiovascular System during exercise. According to Freeman et al. (J. V. Freeman, Dewey, Hadley, Myers, \& Froelicher, 2006) our results are supported by two points: a) the controlling capacity of the cardiovascular functions by the ANS highlighting its role over the cardiac frequency, the myocardium contractile force, vasoconstriction and vasodilation, contraction and relaxation of smooth muscle in several organs and gland secretion; and b) the relationship that the ANS has with the ventilatory function during exercise, given the functions that autonomic afferent fibers have innervating carotid and aortic baroreceptors and controlling respiratory activity (along with cardiac frequency and arterial pressure). According to the previously mentioned relationships between the systems, research reports changes in the autonomic function, sympathetic as well as parasympathetic when increasing exercise intensity (Yamamoto, Hughson, \& Peterson, 1991). An example of this is what Cottin et al. (Cottin, Papelier, \& Escourrou, 1999) and Nakamura et al. (Nakamura, Yamamoto, \& Muraoka, 1993) have described, an increase in the sympathetic activity as the intensity of exercise increases. This is mainly caused by an inhibition of the parasympathetic activities at intensities under $50 \%$ of the $\mathrm{VO}_{2 \max }$. On the other hand, at under greater intensities, the change in the autonomic balance is due to a considerable increase in the sympathetic discharge (Amara \& Wolfe, 1998). The interrelationship between muscle activity characteristic of physical activity and the cardiovascular response is given by the change in the autonomic balance during exercise. At the same time, it depends upon different stimuli which present themselves as muscular metabolic activity increases, and the corresponding increase of metabolites such as lactate and $\mathrm{H}^{+}$(Ansorge et al., 2002). The modification in these metabolite concentrations would cause the stimulation of type III and IV efferent nerve endings located in the muscle (Iellamo, 2001) and consequently an activation of the SNS. This mechanism is known as metaboreflex, which has demonstrated its participation in the regulation of the activity of the sinus node during exercise (Iellamo et al., 1999).

Furthermore, our results are aligned with previous studies of Cottin et al. (Cottin, Leprêtre, \& Lopes, 2006; Cottin et al., 2007) and Karapetian et al. (Karapetian et al., 2008). Both studies have shown a relation between HRV modifications during exercise and AAMT. However, the main difference with our study is given by the physical-mathematical treatment of the HRV as well as the type of exercise performed by our participants. In the study of Cottin et al. (Cottin et al., 2006), 11 trained subjects were studied by the application of an exercise protocol in a cyclergometer. The authors determined to analyze the HRV through a Fourier fast transformed and used the product of the high frequency component and its peak, comparing the behavior of this product with the apparition of the ventilatory threshold 1 and the VT2 (relating them through the power in each exercise phase). In a later research by the same authors (Cottin et al., 2007), this relationship was analyzed in an incremental maximal protocol, performed in a treadmill in 12 subjects. However, a spectro-temporal analysis of the HRV was used through the Wigner - Ville distribution to determine the spectral components of the HRV (HF and HF peak). The results of both studies do not show significant differences between velocities to which changes occur in the product of the HF component and its peak with ventilatory thresholds, significant correlations between them are also found. Similar results are found by Karapetian (Karapetian et al., 2008) who studied 24 healthy adults in an incremental exercise test using the temporal methodology to study the HRV. The results show that there is no significant difference between the HRV threshold point with the VT as well as the LT, and that there is a significant correlation between both thresholds.

This investigation confirms previous results regarding the relationship between the VT2 and the changes in the behavior of the HRV in a maximal incremental exercise. The importance of our study however is based on the use of the DWT as an analysis method for the HRV which is different to what it has been previously used by other researchers, who have used the Fourier fast transformed or a temporal analysis to determine the changes in the HRV. In this context, it is important to highlight the limitations that the FFT presents in its capability to identify the changes in the HRV in conditions were the signal does not present stationary characteristics. It is also worth to highlight the virtues of the DWT in the analysis of the HRV in this type of signals, which has been considered by different authors (Elghozi et al., 
2001; Hossen et al., 2012; Johansson, 2009) as one of the best tools to identify spectral changes of the signal and to detect changes in amplitude and frequency.

\section{Conclusions}

Our results show the AAMT can be obtained through a relatively simple and low cost method in athletes as well as nonathletes. This would allow the possibility to obtain higher quality and more precise information for the control and prescription of exercise and training. Looking forward to a greater application of this method to identify the AAMT and the enhancement of its role in prescription and control of physical exercise, we consider important to apply similar study protocols in populations with associated pathologies. We concluded that in athletes as well as non-athletes the aerobic-anaerobic metabolic transition (i.e. VT2) can be determined through the HRVT in a maximal incremental intensity test using a DWT analysis.

\section{Acknowledgements}

The authors declare no conflicts of interest.

DL acknowledges partial financial support from Basal Program Center for Development of Nanoscience and Nanotechnology (CEDENNA) and UTA-project 8750-12.

\section{References}

Accurso, V., Shamsuzzaman, A. S. M., \& Somers, V. K. (2001). Rhythms, rhymes, and reasons-spectral oscillations in neural cardiovascular control. Auton Neurosci, 90(1), 41-46.

Amara, C. E., \& Wolfe, L. A. (1998). Reliability of noninvasive methods to measure cardiac autonomic function. Can $J$ Appl Physiol, 23(4), 396-408.

Ansorge, E. J., Shah, S. H., Augustyniak, R. A., Rossi, N. F., Collins, H. L., \& O'Leary, D. S. (2002). Muscle metaboreflex control of coronary blood flow. Am J Physiol Heart Circ Physiol, 283(2), H526-H532.

Aubert, A. E., Seps, B., \& Beckers, F. (2003). Heart rate variability in athletes. Sports Med, 33(12), 889-919.

Belova, N. Y., Mihaylov, S. V., \& Piryova, B. G. (2007). Wavelet transform: A better approach for the evaluation of instantaneous changes in heart rate variability. Auton Neurosci, 131(1), 107-122.

Binder, R. K., Wonisch, M., Corra, U., Cohen-Solal, A., Vanhees, L., Saner, H., \& Schmid, J.-P. (2008). Methodological approach to the first and second lactate threshold in incremental cardiopulmonary exercise testing. Eur J Cardiovasc Prev Rehabi, 15(6), 726-734. http://dx.doi.org/10.1097/HJR.0b013e328304fed4.

Cohen, A. (1994). Ten Lectures on Wavelets, CBMS-NSF Regional Conference Series in Applied Mathematics, Vol. 61, I. Daubechies, SIAM, 1992, xix+ 357 pp. Journal of Approximation Theory, 78(3), 460-461.

Cottin, F., Leprêtre, P. M., \& Lopes, P. (2006). Assessment of ventilatory thresholds from heart rate variability in welltrained subjects during cycling. Int J Sports Med, 27(12), 959-967

Cottin, F., Médigue, C., Lopes, P., Leprêtre, P. M., Heubert, R., \& Billat, V. (2007). Ventilatory thresholds assessment from heart rate variability during an incremental exhaustive running test. Int J Sports Med, 28(4), 287-294.

Cottin, F., Papelier, Y., \& Escourrou, P. (1999). Effects of exercise load and breathing frequency on heart rate and blood pressure variability during dynamic exercise. Int J Sports Med, 20(4), 232-238.

De la Cruz Torres, B., López, C. L., \& Orellana, J. N. (2008). Analysis of heart rate variability at rest and during aerobic exercise: a study in healthy people and cardiac patients. $B r \quad J$ Sports Med, 42(9), 715-720. http://dx.doi.org/10.1136/bjsm.2007.043646.

Dewey, F. E., Freeman, J. V., Engel, G., Oviedo, R., Abrol, N., Ahmed, N., . . . Froelicher, V. F. (2007). Novel predictor of prognosis from exercise stress testing: heart rate variability response to the exercise treadmill test. $\mathrm{Am}$ Heart J, 153(2), 281-288.

Drago, S., Bergerone, S., Anselmino, M., Varalda, P. G., Cascio, B., Palumbo, L., . . Trevi, P. G. (2007). Depression in patients with acute myocardial infarction: influence on autonomic nervous system and prognostic role. Results of a fiveyear follow-up study. Int $J$ Cardiol, 115(1), 46-51.

Elghozi, J.-L., Girard, A., \& Laude, D. (2001). Effects of drugs on the autonomic control of short-term heart rate variability. Auton Neurosci, 90(1), 116-121.

Freeman, J. V., Dewey, F. E., Hadley, D. M., Myers, J., \& Froelicher, V. F. (2006). Autonomic nervous system interaction with the cardiovascular system during exercise. Prog Cardiovasc Dis, 48(5), 342-362.

Freeman, R. (2006). Assessment of cardiovascular autonomic function. Clin Neurophysiol, 117(4), 716-730.

Hossen, A., Jaju, D., Al-Ghunaimi, B., Al-Faqeer, B., Al-Yahyai, T., Hassan, M. O., \& Al-Abri, M. (2012). Classification of sleep apnea using wavelet-based spectral analysis of heart rate variability. Technol Health Care, 21(4), 291-303. http://dx.doi.org/10.3233/THC-130724. 
Iellamo, F. (2001). Neural mechanisms of cardiovascular regulation during exercise. Auton Neurosci, 90(1), 66-75.

Iellamo, F., Pizzinelli, P., Massaro, M., Raimondi, G., Peruzzi, G., \& Legramante, J. M. (1999). Muscle metaboreflex contribution to sinus node regulation during static exercise Insights from spectral analysis of heart rate variability. Circulation, 100(1), 27-32.

Johansson, B. (2009). Effects of Functional Water on Heart Rate, Heart Rate Variability, and Salivary Immunoglobulin A in Healthy Humans: A Pilot Study. J Altern Complement Med, 15(8), 871-877. http://dx.doi.org/10.1089/acm.2008.0336.

Karapetian, G. K., Engels, H. J., \& Gretebeck, R. J. (2008). Use of heart rate variability to estimate LT and VT. Int J Sports Med, 29(8), 652-657. http://dx.doi.org/10.1055/s-2007-989423.

Karvonen, M. J. (1957). The effects of training on heart rate. A longitudinal study. Ann Ned Exp Biol Fenn, 35, $307-$ 315.

Knoepfli, B., Riddell, M. C., Ganzoni, E., Burki, A., Villiger, B., \& Von Duvillard, S. P. (2004). Off seasonal and preseasonal assessment of circulating energy sources during prolonged running at the anaerobic threshold in competitive triathletes. Br J Sports Med, 38(4), 402-407.

Manso, J. M. G. (2013). Aplicación de la variabilidad de la frecuencia cardiaca al control del entrenamiento deportivo: análisis en modo frecuencia. Arch Med Deporte, 30(1), 43-51.

Marks, B. L., \& Lightfoot, J. T. (1999). Reproducibility of resting heart rate variability with short sampling periods. Can J Appl Physiol, 24(4), 337-348.

Meyer, T., Lucia, A., \& Earnest, C. P. (2005). Submaximal Parameters \pm Theory and Application. Int J Sports Med, 26, $1-11$.

Nakamura, Y., Yamamoto, Y., \& Muraoka, I. (1993). Autonomic control of heart rate during physical exercise and fractal dimension of heart rate variability. J Appl Physiol, 74(2), 875-881.

Pichot, V., Gaspoz, J.-M., Molliex, S., Antoniadis, A., Busso, T., Roche, F., . . Barthélémy, J.-C. (1999). Wavelet transform to quantify heart rate variability and to assess its instantaneous changes. J Appl Physiol, 86(3), 1081-1091.

Sartor, F., Vailati, E., Valsecchi, V., Vailati, F., \& La Torre, A. (2013). Heart rate variability reflects training load and psychophysiological status in young elite gymnasts. $J$ Strength Cond Res, 27(10), 2782-2790. http://dx.doi.org/10.1519/JSC.0b013e31828783cc.

Sone, R., Yamazaki, F., Fujii, N., Fukuoka, Y., \& Ikegami, H. (1996). Respiratory variability in RR interval during sinusoidal exercise. Eur J Appl Physiol Occup Physiol, 75(1), 39-46.

Thayer, J. F., \& Sternberg, E. (2006). Beyond heart rate variability. Ann N Y Acad Sci, 1088(1), 361-372.

Verlinde, D., Beckers, F., Ramaekers, D., \& Aubert, A. E. (2001). Wavelet decomposition analysis of heart rate variability in aerobic athletes. Auton Neurosci, 90(1), 138-141.

Yamamoto, Y., Hughson, R. L., \& Peterson, J. C. (1991). Autonomic control of heart rate during exercise studied by heart rate variability spectral analysis. J Appl Physiol, 71(3), 1136-1142. 